\section{Safety of leukoreduced, cytomegalovirus (CMV)-untested components in CMV-negative allogeneic human progenitor cell transplant recipients}

\begin{abstract}
Transfusion-transmitted cytomegalovirus (TT-CMV) infection can lead to significant morbidity and mortality in CMV-negative (CMV-N) hematopoietic progenitor cell (HPC) transplant patients. In 1995, Bowden and colleagues $^{1,2}$ demonstrated the efficacy of leukoreduced components to reduce TT-CMV in most high-risk populations, although there remained safety concerns in CMV-N allogeneic HPC transplant recipients. As a result, several transplant programs recommended both leukoreduced and CMV-N components for CMV-N allogeneic HPC patients receiving transplants from CMV-N donors (CMV $\left.{ }^{\text {neg/neg }}\right)^{2}$ A more recent study, however, has challenged the clinical benefit of requiring CMV-N, in addition to leukoreduction, in $\mathrm{CMV}^{\text {neg/neg }} \mathrm{HPC}$ patients. In a 10-year study, Thiele and colleagues ${ }^{3}$ found no cases of TT-CMV in $23 \mathrm{CMV}^{\text {neg/neg }} \mathrm{HPC}$ patients transfused with $1847 \mathrm{CMV}$ untested (CMV-U), leukoreduced components. We would like to share our institution's experience in $100 \mathrm{CMV}^{\text {neg/neg }}$ allogeneic HPC patients transfused with 6465 CMV-N and CMV-U cellular components.
\end{abstract}

Before July 2006, the University of Michigan provided, when available, CMV-N components to all $\mathrm{CMV}^{\text {neg/neg }}$ HPC transplant recipients. A preliminary 12-month retrospective review showed that $52 \%(14 / 27$, 2004) had received leukoreduced, CMV-U blood components due to shortages in CMV-N products, with no cases of TT-CMV $^{4}$ As a result, the transfusion policy was changed in mid-2006 to provide leukoreduced, CMV-U products for all HPC patients, regardless of pretransplant CMV status. For quality assurance, we monitored the CMV conversion rate over a 36-month period (January 2005 to December 2007) covering an 18-month period before and after the change in transfusion policy (Table 1). Per institutional practice, CMV-N patients were screened for CMV IgG every 1 to 2 months pretransplant, followed by regular testing for CMV nucleic acid testing (NAT) after transplant. The minimum posttransplant follow-up was 12 months. All blood products were provided by Southeast Michigan American Red Cross (Detroit, MI) and were leukoreduced after storage (Leukotrap RC system, Pall Corp., Port Washington, CA).

As shown in Table 1, 100 patients were available for analysis and included both adult and pediatric patients. All patients were CMV-N before transplant, received a $\mathrm{CMV}^{\text {neg/neg }}$ allogeneic HPC transplant, and underwent weekly posttransplant CMV NAT monitoring. Except for sex, there were no significant differences in patient demographics, transplant type, or transfusion support in the two study cohorts. In the CMV-N policy period, only $11 \%$ to $15 \%$ of cellular components were CMV-N, with most patients receiving a mix of CMV-N and CMV-U. Only five patients received $100 \%$ CMV-N products. All five patients had low transfusion needs, requiring 2 to 10 red blood cell (RBC) and two to three platelet (PLT) transfusions.

Two adult male patients had a single positive test for CMV IgG at 3 and 5 weeks after transplant, respectively (Table 1). Both patients tested negative for CMV IgM and CMV NAT and had no evidence of clinical CMV infection. Each patient received between 42 and $45 \mathrm{CMV}$-U cellular components in the weeks before seroconversion: neither patient had received intravenous immune globulin before CMV IgG testing. There were no CMV seroconversions in the CMV-U period. The overall CMV IgG seroconversion rate was $2 \%$ per patient and $0.03 \%$ per unit, which is comparable to the findings by Bowden and coworkers ${ }^{1}(2.4 \%$ per patient, $0.023 \%$ per component). The rate of confirmed TT-CMV was $0 \%$, consistent with the study by Thiele and coworkers ${ }^{3}$ and lower than that reported by Wu and coworkers ${ }^{5}(6.5 \%$ per patient, $0.23 \%$ per CMV-positive component). As discussed by Thiele and Wu, the CMV IgG detected in our patients likely represents passive antibody from recent transfusions. ${ }^{3,5}$

Our findings confirm those of Thiele and affirm the equivalent safety of $\mathrm{CMV}-\mathrm{U}$, leukoreduced components in $\mathrm{CMV}^{\text {neg/neg }}$ allogeneic HPC patients. ${ }^{3}$ The absence of clinical TT-CMV infection in our study and that by Thiele and coworkers, ${ }^{3}$ despite the combined transfusion of nearly $8000 \mathrm{CMV}-\mathrm{U}$, leukoreduced components, contradicts sentiments from a past multivariate analysis, which advocated continued provision of CMV-N and leukoreduced components for $\mathrm{CMV}^{\text {neg }}$ transplant patients. ${ }^{6}$ The improved safety of CMV-N over CMV-U, leukoreduced is also not supported by a recent large prospective study of 34,000 blood donors. Ziemann and colleagues ${ }^{7}$ found CMV viremia only among newly seroconverted donors and a few CMV-N donors, arguing that CMV-N components may present the higher risk of TT-CMV due to passive transfusion of free CMV DNA. In summary, policies stipulating leukoreduced, CMV-N components in $\mathrm{CMV}^{\text {neg/neg }}$ allogeneic HPC patients do not confer additional safety and are limited by product shortages and significant transfusion support required by many allogeneic HPC patients.

\section{ACKNOWLEDGMENTS}

This work was partially supported by the Robert Woods Johnson Clinical Scholars Program (TN, University of Michigan, 2009). 


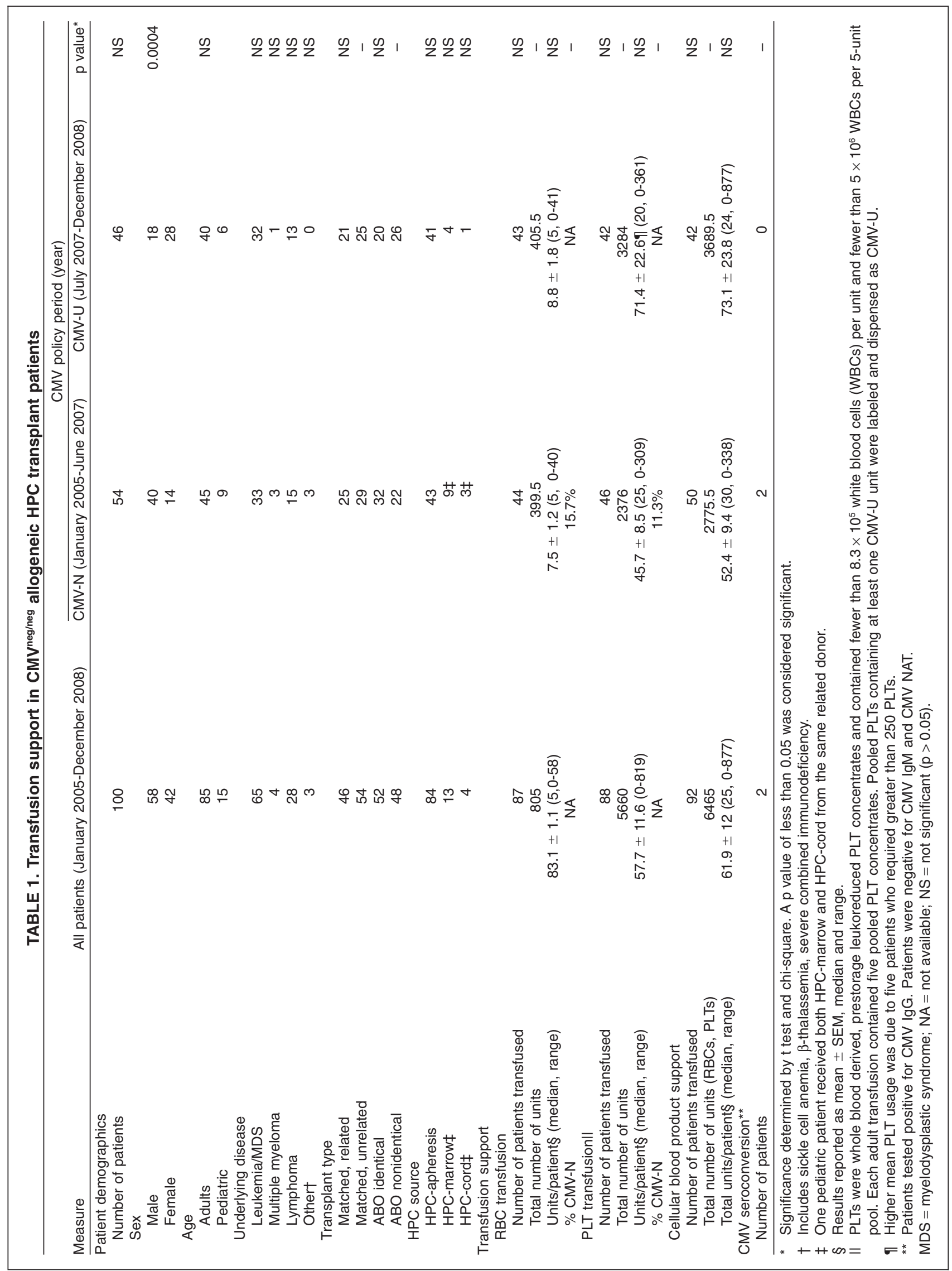




\section{CONFLICT OF INTEREST}

The authors certify that they have no affiliation with or financial involvement in any organization or entity with a direct financial interest in the subject matter or materials discussed in this manuscript.

Tammon Nash, MD, MS Michigan Blood

Grand Rapids, $M I$

Sandra Hoffmann, MT(ASCP)SBB Suzanne Butch, MT(ASCP)SBB Robertson Davenport, MD Laura Cooling, MD, MS e-mail:lcooling@med.umich.edu Department of Pathology University of Michigan

Ann Arbor, $M I$

\section{REFERENCES}

1. Bowden RA, Slichter SJ, Sayers M, Weisdorf D, Cays M, Schoch G, Banaji M, Haake R, Welk K, Fisher L, McCullough J, Miller W. A comparison of filtered leukocyte-reduced and cytomegalovirus (CMV) seronegative blood products for the prevention of transfusion-associated CMV infection after marrow transplant. Blood 1995;86:3598-603.

2. Smith D, Lu Q, Yuan S, Goldfinger D, Fernando LP, Ziman EA. Survey of current practice for prevention of transfusion-transmitted cytomegalovirus in the United States: leucoreduction vs. cytomegalovirus-seronegative. Vox Sang 2010;98:29-36.

3. Thiele T, Kruger W, Zimmermann K, Ittermann T, Wessel A, Steinmetz I, Kolken G, Greinacher A. Transmission of cytomegalovirus (CMV) infection by leukoreduced blood products not tested for CMV antibodies: a single-center prospective study in high-risk patients undergoing allogeneic hematopoietic stem cell transplantation. Transfusion 2011;51:2620-6.

4. Hoffmann S, Herrst M, Butch S, Cooling L. Prestorage leukocyte-reduced, CMV untested blood components are safe for use in CMV negative allogeneic bone marrow transplant recipients [abstract]. Transfusion 2007;47:212-3.

5. Wu Y, Zou S, Cable R, Korsey K, Tang Y, Hapip CA, Melmed R, Trouern-Trend J, Wang JH, Champion M, Fang C, Dodd R. Direct assessment of cytomegalovirus transfusiontransmitted risks after universal leukoreduction. Transfusion 2010;50:776-86.

6. Vamvakas EC. Is white blood cell reduction equivalent to antibody screening in preventing transmission of cytomegalovirus by transfusion? A review of the literature and meta-analysis. Tranfus Med Rev 2005;19:181-99.

7. Ziemann M, Krueger S, Maier AB, Unmack A, Goerg S, Hennig H. High prevalence of cytomegalovirus DNA in plasma samples of blood donors in connection with seroconversion. Transfusion 2007;47:1972-83.

\section{Does modern combat still need fresh whole blood transfusions?}

In combat, massive blood loss of military as well as of civilians is a major cause of death. Having blood available in the military theater at all times is therefore of vital importance. Fresh whole blood (FWB) has been used repeatedly by the US military to resuscitate severely bleeding trauma patients. ${ }^{1} \mathrm{FWB}$ is blood that is donated by military and hospital personnel on site and stored for less than 24 hours in citrate-phosphate-dextrose solution at room temperature. The US military supports transfusion of FWB either when standard blood components are not available or if transfusion of the available blood components are not adequately correcting life-threatening bleeding. Although warm FWB has the theoretical advantage of supplying all the appropriate blood components with maximal functionality, usage of untested FWB proposes a risk of infectious disease transmission and bacterial contamination as well as logistic difficulties in obtaining identical match donors at the right time. Transfusion of FWB has also been associated with a higher incidence of adverse reactions through the presence of white blood cells (WBCs). These reactions include febrile nonhemolytic transfusion reactions, human leukocyte antigen alloimmunization and transfusion-associated graft-versus-host disease (TA-GVHD). ${ }^{2}$ Hence, in a recent article by Gilstad and colleagues ${ }^{3}$ clinical symptoms of TA-GVHD were observed in a trauma patient who was resuscitated with FWB.

Usage of FWB is often endorsed because of the potential damaging effects of prolonged refrigerated stored RBCs. Yet, frozen storage of RBCs at ultralow temperatures halts the cellular metabolism and subsequently prevents the progressive RBC deterioration that has been linked to adverse clinical outcome. ${ }^{4}$ During the years, frozen blood components have become more utilizable. Notably, implementation of frozen platelets (PLTs) and fresh-frozen plasma in transfusion medicine has abandoned the need for FWB usage. Consequently, in 2001 the Dutch military blood bank eliminated the use of FWB on site and implemented the routine usage of universal frozen blood components. ${ }^{5,6}$ In this regard, leukoreduced blood components are frozen at $-80^{\circ} \mathrm{C}$ within 24 hours after collection. After thaw, RBCs, fresh-frozen plasma, and PLTs can be utilized up to 14 days, 7 days, and 6 hours, respectively. The Dutch military has demonstrated that frozen blood components can provide an adequate blood resource, even when standard blood components cannot be replenished on time. This allows for a better inventory control, especially in remote or primitive locations.

Usage of frozen blood components has the advantage that it is safe. This is because blood components have been collected and preserved under standard conditions 